$P^{\prime}$ takes the place of $P$, and the new elements are functions of the original elements. $\quad \mu$ is determined from the condition that the new series must be of the same general form as the old. If, in addition, $x$ be replaced by $1 / x$ another series is obtained. From these two new series, by proper substitution of the new derived elements, are obtained, almost by inspection, the twentyfour different series ordinarily given in works on differential equations.

Edwin S. Crawley,

University of Pennsyluania.

Secretary.

\title{
SECOND REPORT ON RECENT PROGRESS IN THE THEORY OF GROUPS OF FINITE ORDER.
}

BY PROFESSOR G. A. MILLER.

(Read before Section A of the American Association for the Advancement of Science, Pittsburg, July 2, 1902.)

THE main extensive treatments of this theory which have appeared during the four years since my first report was presented before this Section are: The articles in volume I of the Encyklopädie der mathematischen Wissenschaften on "Endliche discrete Gruppen," "Galois'sche Theorie mit Anwendung," and "Endliche Gruppen linearer Substitutionen," by Burkhardt, Hölder, and Wiman respectively; Weber, Lehrbuch der Algebra, second edition, volume 2, 1899; Bianchi, Lezioni sulla teoria dei gruppi di sostituzioni, 1899 ;* Echegaray, Lecciones sobre resolucion de ecuaciones y teoria de ecuaciones, $1899 ; \dagger$ Netto, Vorlesungen über Algebra, volume 2, 1900 ; Pierpont, "Galois theory of algebraic equations," $\$ 1900$; Dickson, Linear groups, 1901; Burnside and Panton, Theory of equations, volume $2,1901$.

In the present, as in my first report, it is my intention to avoid, as far as practicable, the consideration of those recent advances which have received considerable attention in these

* Printed edition of the work lithographed in 1897.

+ Reviewed in L' Enseignement Mathématique, vol. 2 (1900), p. 227.

$\ddagger$ Annals of Math., $2 \mathrm{~d}$ series, vols. 1 and 2 . 
articles and treatises, and to devote most of the space to a few recent developments which seem to offer inviting fields of investigation. It is hoped that this report will soon be supplemented in some directions, like the first report the usefulness of which was greatly extended by Professor Dickson.*

For several years Dr. Steinitz, of Charlottenberg, has had under consideration a report on the theory of groups of finite order for the Deutsche Mathematiker-Vereinigung. In a recent letter he expressed the hope of having this report ready for the next meeting of the Vereinigung, which will be held at Karlsbad during the coming September in connection with Section I of the Gesellschaft Deutscher Naturforscher und Aerzte. Dr. Easton, of the University of Pennsylvania, has prepared a bibliography of the theory of substitutions together with a collection of theorems and definitions, which is now in press.

\section{§1. Abstract Groups.}

If the three numbers $l, m, n$ are the orders of three operators one of which is a product of the other two, it is well known that the group generated by any two of these operators is completely determined by $l, m, n$ provided at least two of these numbers are 2 ; or one is 2 ; another 3 , while the third is 3,4 , or 5 . The groups which may be defined in this way, together with those which are cyclic, are known as the groups of genus zero, and have received a great deal of attention as they are the groups of the rotations into themselves of the regular solids and hence enter prominently into many geometric considerations.

It has recently been proved $\uparrow$ that three operators $L, M, N$ whose orders are fixed numbers $l, m, n$ respectively can always be so selected as to generate any one of an infinite system of groups of finite order whenever each of these fixed numbers exceeds unity and the three do not satisfy one of the special conditions mentioned in the preceding paragraph. The proof of this theorem is based upon the fact that it is always possible to find three operators such that one of them is a product of the other two and that their orders are the three arbitrary numbers $l, m, n$. Both of these theorems were proved by means of sub-

* Dickson, " Report on the recent progress in the theory of linear groups," Bulletin, vol. 6 (1899), p. 13.

$\dagger$ Amer. Jour. of Math., vol. 24 (1902), p. 96 ; cf. Dyck, Math. Annalen, vol. 20 (1882), p. 34 . 
stitutions. It would appear desirable that more abstract proofs should be given, especially since the published proofs are based upon considerations which are not commonly employed.

The infinite systems of groups which belong to a particular set of values of $l, m, n$ have received very little attention except in the three cases where these numbers are such as to give rise to groups of genus one. These cases have been studied geometrically by Dyck* and Burnside. $\dagger$ Recently they have been studied from the purely analytic standpoint and some interesting common properties have been found. $\ddagger$ Perhaps the most important of these is the fact that all of them contain an abelian commutator subgroup.

The groups of genus one which are not included in the three cases mentioned in the preceding paragraph are those which may be generated by three operators of order two whose continued product is also of order two, but which cannot be generated by two operators of order two. All of these groups may be constructed by adding to any abelian group $A$, having just two independent generators, an operator of order two which transforms each operator of $A$ into its inverse. In this way a group is obtained whose order is twice the order of $A$ and which contains only operators of order two besides those of $A$. This is the simplest category of groups of genus one and has many properties in common with the dihedral rotation groups. It may be observed that none of these groups can be generated by less than three operators. This category includes only one abelian group, viz., the group of order 8 and of type $(1,1,1)$.

From what precedes it is clear that little is known about the groups generated by the two operators $L$ and $M$ when the three numbers $l, m, n$ are given. A closely related problem which has received but little attention is the study of the groups in which the order of every operator is a divisor of a fixed number $k$. The simplest case, viz., when $k=2$, has been completely solved. $\S$ There is just one such group of order $2^{a}$ for every value of $\alpha$. This is the only value of $k$ for which all the possible

* Loc. cit.

+ Theory of groups of finite order, 1897, p. 293.

tQuar. Jour. of Math., vol. 33 (1901), p. 76 ; Annals of Math., vol. 3 (1901), p. 40 ; The case when the groups are generated by two operators of order 4 whose product is of order 2 was considered by Manning in a paper read at the first meeting of the San Francisco section of the Amer. Math. Soc., May, 1902.

§Quar. Jour. of Math., vol. 28 (1896), p. 208. 
groups are abelian. When $k=3$ it is very easy to prove that all the conjugate operators are commutative. Moreover, it is evident that this property is not common to all the groups for any value of $k$ greater than 3 .

Recently Burnside has made a study of all the possible groups when $k=3$, and also of the special case when $k=4$ and the groups have but two generators.* The more general case when $k$ is any prime $p$ seems to present considerable difficulty. The order of such a group is evidently a power of $p$. In the special case when there is an abelian subgroup whose order is the order of the entire group divided by $p$ it is not difficult to determine all the possible groups. $t$ Even for $p^{5}, p>3$, there are groups which contain only operators of order $p$ without containing the abelian group of order $p^{4}$.

The questions considered in the preceding two paragraphs may be regarded as special cases of the problem to determine all the groups which are conformal with systems of abelian groups. If two abelian groups are conformal, $i$. $e$, if they contain the same number of operators of each order, they cannot be distinct; but with respect to the non-abelian groups the matter is entirely different. Recently all the possible abelian groups which are conformal with non-abelian groups have been determined, $\ddagger$ but the total number of non-abelian groups which are conformal with fixed types of abelian groups are known in only a few cases. For instance, it is known that there is only one non-abelian group which is conformal with the abelian group of order $p^{m}$ and of type $(m-1,1)$. The only other types of order $p^{m}$ for which all the conformal non-abelian groups are known are $(m-2,2)$ and $(m-2,1,1)$.

If $p^{a}$ is the highest power of the prime number $p$ which divides the order of a group then the group must contain $1+k p$ conjugate subgroups of order $p^{\alpha}$ according to Sylow's theorem. Moreover, it is known that there are groups for all values of $p$ when $k$ is either 0 or 1 . Burnside has recently examined the cases when $k$ is either 2 or 4 and found that in both of these cases the possible values of $p$ are greatly restricted. His results are, when $k=2$, then $1+2 p$ is either a prime or a power of 3 ; and when $k=4$, then $1+4 p$ is either a prime, 9 ,

* Burnside, Quar. Jour. of Math., vol. 33 (1902), p. 230.

† Bulletin, vol. 8 (1902), p. 39.

+ Bulletin, vol. 8 (1902), p. 154.

\& Loc. cit., p. 206. 
or a power of 5 . The case when $k=3$ seems to present greater difficulties but it is easy to see that there are also limitations on the form of $p$ for this value of $k$.*

Most of these advances in the theory of abstract groups have been made by means of substitution groups. When this method is employed comparatively little use is made of definitions, as all the steps can be conveniently illustrated by means of concrete examples. For some purposes it is however desirable to proceed more abstractly. This seems to be especially the case in a systematic presentation of the subject. Hence it becomes desirable that the definitions should be so formulated as to be as useful as possible. It is also desirable to avoid redundancies as these mar the beauty of the presentation. Recently Dr. Huntington has pointed out redundancies in the definitions usually given and has formulated definitions which do not involve these objectionable features. $\dagger$ Professor Moore has also presented a note on this subject. $f$

Scarpis has recently considered some properties of commutators and of commutator subgroups. He proves by a method which differs somewhat from the one employed earlier, that the important question of solvability can readily be decided by means of commutator subgroups. $\$$ Although he gave a general reference to the earlier article in which this theorem is proved, yet one would naturally infer in reading his article that he considered the theorem as new. One might also question the propriety of naming these groups after Dedekind, since he published very little in regard to them, and, moreover, was not the first to make their properties known.\|

The interesting question whether every operator of a commutator subgroup is a commutator has been answered in the negative in volume 2 , page 133 , of the second edition of Weber's Algebra. Dr. Fite has found some conditions which are sufficient for the existence of such operators, and has published a group which satisfies these conditions. $\uparrow$ Wundt proved a very interesting theorem in regard to the special class of soluble groups which possess a principal series of composition such that

* Burnside, Messenger of Mathematics, vol. 31 (1901), p. 77.

† Huntington, BuLletin, vol. 8 (1902), p. 296.

t BuLletin, vol. 8 (1902), p. 373.

$\S$ Scarpis, Giornale de Mutematiche, vol. 39 (1901), p. 376 ; ef. Bulletin, vol. 6 (1899), p. 105.

|| (Yf. Frobenius, Berliner Sitzungsberichte, 1896, p. 1348 ; Cantor, Geschichte der Mathematik, vol. 2 (1900), p. 811.

đI Bulletin, vol. 6 (1900), p. 181. 
all of the corresponding factor groups are of prime order. Whenever this condition is satisfied he proved that the commutator subgroup must be the direct product of groups whose orders are powers of primes.* This proof is partly based upon the fact that every group contains an invariant subgroup (which may be the identity) which is the direct product of groups whose orders are powers of primes and includes all other invariant subgroups that have this property.

The most extensive work on the enumeration of abstract groups which has appeared during the last few years seems to be the Enumeration des groupes d'opérations d'ordre donné, 1901, 128 p., lith., by Le Vavasseur. This work, which is published by Hermann, Paris, was noticed by the writer too lately to permit an examination before writing this report. The same author has recently published less extensive enumerations of the groups of order $p^{2} q^{2}$ and also those of order $16 p, p$ and $q$ being primes and $p$ being odd. $\dagger$ Western has considered the groups of order $p^{3} q, \div$ but no one seems to have determined all the groups in the remaining case where there are four prime factors, viz., when the order is $p^{2} q r$. The enumeration of groups of low orders has been extended to those of order $64 . \S$

Dickson has embodied a large number of the results of his extensive investigations in his Linear Groups. The last chapter of this work is devoted to a summary of known systems of simple groups. It is observed that two of these systems involve simple groups of the same order that are not isomorphic. The object of some of his more recent papers is to show that several branches of group theory may be correlated by means of transformations in a given domain of rationality. The special simple group of order 504 has been defined more abstractly by Burnside in the Mathematische Annalen, volume 52 (1899), page 174. Fricke considers this group from a different standpoint in the same volume, page 321.

In a memoir published in Bihang till Kongl. Svenska Vetenskaps Akademiens Handlingar, volume 25 (1900), Wiman determined all the subgroups of a doubly infinite system of simple groups. The article begins with a study of the Galois imaginaries and the groups of finite order defined by congruences.

\footnotetext{
* Wundt, Math. Annalen, vol. 55 (1901), p. 479.

† La Vavasseur, Comptes rendus, Paris, vol. 128 (1899), p. 1152 ; vol. 129, p. 26.

¥Western, Proc. of the London Math. Soc., vol. 30, p. 209.

$\S$ Quar. Jour. of Math., vol. 30 (1898), p. 243.
} 
Bauer, in a series of articles published in Nouvelles Annales,* proved a number of theorems relating to the number of subgroups of particular orders that can occur in a group whose order is known. Most of these theorems are based upon those proved by Frobenius in the Berliner Sitzungsberichte of 1895 .

While some questions in regard to abelian groups, for instance those which relate to the number and the types of groups of a fixed order, have been completely solved yet there is a large number of others which have received but little attention. Steinitz has recently considered one of the latter, viz., the determination of how many times a group $\gamma$ is contained in the product $a \cdot \beta$ of two abelian groups. In other words, to determine the number of subgroups $\alpha^{\prime}$ such that $\alpha^{\prime}$ is isomorphic with $\alpha$ and $\gamma / \alpha^{\prime}$ is isomorphic with $\beta$. Representing this number by $t(\gamma ; \alpha, \beta)$ Steinitz investigated some of the properties of this function and determined all these groups when $\beta$ is cyclic or has just two independent generators. $\dagger$

On the occasion of the jubilee of Sir G. G. Stokes, Burnside presented a brief memoir on the groups of even order which contain no operator of even order except those of order 2. He found that these groups may be divided into three distinct classes which are represented by the dihedral, tetrahedral, and the icosahedral rotation groups. In a more recent paper communicated to the London Mathematical Society he considers the groups in which every two conjugate operators are permutable and proves that the necessary and sufficient condition that the order of such a group is finite is that the generators are of finite orders. The case when the order is a power of 3 is exceptional. +

\section{§ 2. HoLOMORPHisms.}

A simple isomorphism of a group $G$ with itself has been called a holomorphism or an automorphism of $G$. $\S$ The totality of the holomorphisms of $G$ correspond to a group $I$ known as the group of isomorphisms. When $G$ is transformed by its own operators one or more holomorphisms are obtained. The totality of the holomorphisms that can be obtained in this way correspond to an invariant subgroup $I_{1}$ of $I$, which is known as

* Vol. 19 (1900), pp. 59, 508, 509.

† Steinitz, Jahresbericht der Deutschen Math.-Vereinigung, vol. 9 (1901), p. 80. † Nature, vol. 66 (1902), p. 71.

\&. Annals of Math., vol. 2 (1901), p. 78 ; Frobenius, Berliner Sitzungsberchte, 1901, p. 1324. 
the group of cogredient isomorphisms. When $I$ is abelian, $G$ is said to be metabelian and vice versa. A characteristic property of metabelian groups is that all their commutators are invariant.

The metabelian groups constitute a special case of a category of groups which seems to have been first studied by Ahrens; viz., those in which one arrives at the identity by forming the successive groups of cogredient isomorphisms.* It is easy to prove that all the groups of this category are the direct products of groups whose orders are powers of single prime numbers and vice versa. $f$ Dr. Fite has recently made an extensive study of the properties of metabelian groups and some of his results have been published in the form of abstracts. His memoir on these groups is to appear soon in the Transactions of the American Mathematical Society.

The term metabelian seems appropriate since these groups hare many properties which differ but slightly from those of abelian groups. In the development of their theory it is convenient to make prominent use of the commutator subgroup and the group of cogredient isomorphisms. When the former is cyclic and of order $p^{\alpha}, p$ being any prime, the latter contains an even number of independent generators, and the corresponding metabelian groups are the direct products of abelian groups and a metabelian group of order $p^{m} . \neq$ The Hamiltonian groups constitute a special case of those metabelian groups whose group of cogredient isomorphisms is the four-group. The theory of metabelian groups clearly involves the determination of all the abelian groups which can be groups of cogredient isomorphisms. Most of the results in this direction are found in the memoir mentioned in the preceding paragraph.

A number of important properties of a group are exhibited in its group of isomorphisms. Comparatively little progress has been made in the theory of the simple isomorphisms of a group with itself. One of the most important steps in this direction has been the determination of all the invariant operators of $I$ when $G$ is abelian. These operators correspond to the holomorphisms of $G$ in which every subgroup corresponds to itself. If this condition is satisfied every operator of $G$ corresponds to

* Ahrens, Leipziger Berichte, vol. 49 (1897), p. 616 ; cf. Fite, Bulletrin, vol. 8 (1902), p. 236 .

† Burnside, Theory of groups, 1897, p. 115 ; ef. Loewy, Math. Annalen, vol. 55 (1901), p. 67 .

\$ Bulletin, vol. 7 (1901), p. 86 . 
the same power of itself.* Hence the number of invariant operators of $I$ is the totient of the maximum order of any operator of $G$.

These results have been very materially extended by $\mathrm{Mr}$. Young in his recent article "On the holomorphisms of a group." $\dagger \mathrm{He}$ employs for the first time the convenient term a-holomorphism to denote a simple isomorphism of a group with itself such that every operator corresponds to its $\alpha$ th power, and inquires into the necessary and sufficient condition that a non-abelian group may admit $\alpha$-holomorphisms besides the identical one. He finds that a group cannot admit an $\alpha$-holomorphism unless the $(\alpha-1)$ th power of every operator is invariant. When the order of the group is a power of a prime these $\alpha$-holomorphisms correspond to invariant operators in the group of isomorphisms but it is not always possible to obtain all the invariant operators of the latter group in this manner.

In any holomorphism each operator corresponds to itself multiplied on the right by some operator of the group. The totality of these multiplying operators do not necessarily form a group. For instance, if the symmetric group of order 6 is transformed by one of its operators of order three, the holomorphism will be such that the multiplying operators do not form a group ; but these operators form a group when the holomorphism may be obtained by transforming by an operator of order two. In every holomorphism of an abelian group $A$ these multipliers always form a group $\leftarrow$ and it is frequently convenient to consider the holomorphisms of $A$ from this standpoint.

Not only do the multipliers of the preceding paragraph form a subgroup of $A$ but this subgroup (which may coincide with $A$ ) is also isomorphic with $A$ in every possible simple isomorphism of $A$ with itself. If the order of this subgroup is either $p$ or $2 p$; the resulting holomorphism of $A$ will correspond to an operator of order $p, 2 p$, or $\frac{p-1}{\alpha}(\alpha$ being a divisor of $p-1)$ in the group of isomorphisms $I$ of $A$. If, moreover, each operator of this subgroup corresponds to itself in such a holomorphism, the order of the corresponding operator of $I$ is clearly equal to the order of the largest operator of the subgroup. Very little has been done towards the development of the theory of holomor-

* Transactions Amer. Math. Soc., vol. 2 (1901), p. 260.

† Young, Ibid., vol. $3(1902)$, p. 186.

† Bulletin, vol. 6 (1900), p. 337. 
phisms from this standpoint. It may be possible to find simple conditions which are necessary and sufficient to establish holomorphisms of non-abelian groups in this manner. Such conditions would be of considerable interest.

One of the simplest ways of obtaining a holomorphism of a non-abelian group $G$ is to make each of its operators $A$ correspond to its transform with respect to any one of its operators $B$. In calling attention to a slight error in Burkhardt's article in the Encyklopaedie, Loewy writes this transform $B A B^{-1}$ instead of $B^{-1} A B$. $^{*} \quad$ Moreover Loewy writes $B A^{-1} B^{-1}$ instead of $B A^{-1} B$ as given by Burkhardt. The latter is, however, a very insignificant error; while the former cannot be regarded as an error since some good writers have used $B A B^{-1}$ for the transform of $A$ with respect to $B ; \dagger$ but the majority of writers (including Jordan, Lie, Netto, Weber, Burnside, Bianchi) use the form $B^{-1} A B$. The form used by the minority seems to have decided advantages. For instance, in considering the expression

$$
\begin{aligned}
& (A B)^{n}=A B A B A B A B \ldots \\
& =A B A B^{-1} B^{2} A B^{-2} B^{3} A B^{-3} \ldots
\end{aligned}
$$

it is convenient to call $B A B^{-1}$ the transform of $A$, especially if some of the commutators are not invariant. Although this is not a very important matter yet it would be of some interest to know which of these forms is the most advantageous, especially since transforms are so frequently employed.

If any group $G$ is represented as a regular substitution group there is a totality of substitutions in the same elements as those involved in $G$ which transform $G$ into itself. These form a non-regular group known as the holomorph of $G$. Any one of the largest subgroups which do not involve one of the elements of this holomorph is simply isomorphic with the group of isomorphisms of $G$ and every holomorphism of $G$ can therefore be obtained by transforming $G$ by some operator in such a subgroup. The substance of these remarks is found in my former report, page 245. It is repeated here since Burkhardt's statement on bottom of page 221 of the Encyklopaedie seems to imply that the holomorph of $G$ is also its group of isomorphisms, which is evidently incorrect.

* Loewy, Math. Annalen, vol. 55 (1901), p. 68.

† Dyck, Math. Annalen, vol. 22 (1883), p. 75. The present usage seems to be due to the influence of Jordan ; cf. Hagen, Synopsis der höheren Mathematik, vol. 1 (1891), p. 285 . Cauchy and Serret used the form $B A B^{-1}$, which was called the derivative of $A$ by Betti. 
It is known that cyclic groups are the only abelian groups whose groups of isomorphisms are abelian.* No one seems to have investigated the question whether a non-abelian group can have an abelian group of isomorphisms. If this question should be answered in the negative it will follow that the system of groups which Weber calls the most important example of abelian groups of finite order $\dagger$ is composed of all the possible commutative groups of isomorphisms. Since the group of cogredient isomorphisms of a non-abelian group cannot be cyclic, it follows that a cyclic group cannot be a group of isomorphisms unless its order is of the form $p^{\alpha}(p-1), p$ being an odd prime.

Groups which have the same group of isomorphisms may differ very much from each other. The totality of such groups has been determined in only a few cases while no one seems to have determined all those which have the same group of cogredient isomorphisms. Some of the simplest cases would evidently present very few difficulties. For instance, when the group of cogredient isornorphisms of $G$ is of order $p^{2}$ it must be of type $(1,1)$ and $G$ must be the direct product of abelian groups and a non-abelian group $P$ of order $p^{m}$ whose group of cogredient isomorphisms $I_{1}$ is of order $p^{2}$. Moreover, $P$ contains an abelian subgroup of order $p^{m-1}$ which includes the $p^{m-2}$ invariant operators. $\mathrm{W}$ hen this abelian subgroup is cyclic it is well known that there is only one such group. When it is of type $(1,1,1, \cdots)$ there are clearly three such groups if $p>2$ and $m>3$; one is conformal with the abelian group of type $(1,1,1, \ldots)$ while the others are conformal with the one of type $(2,1,1, \cdots)$.

\section{§3. Substitution Groups.}

Maillet and Burnside have considered the transitive groups of degree $n$ and of class $n-1$. In his thesis entitled, "Recherches sur les substitutions, et en particulier sur les groupes transitifs," Paris, 1892, Maillet divides these groups into two categories. The first of these included all those which contain a regular group of order $n$, while the second included those which do not have this property. He states that in all the particular cases which he has examined there is no primitive group belonging to the second category. In several later articles pub-

* Transactions, vol. 1 (1900), p. 397.

$\dagger$ Weber, Lebrbuch der Algebra, vol. 2 (1899), p. 60. 
lished in the Bulletin of the French Mathematical Society* he extends a number of his results in regard to these groups.

Burnside takes up the same question both in his Theory of Groups and also in an article published in the Proceedings of the London Mathematical Society. $\dagger$ In the latter he proves that every transitive group of degree $n$ and of class $n-1$ contains a regular group of order $n$ whenever $n \equiv 81,000,000$. Recently Frobenius proved $\ddagger$ that this theorem is true for all values of $n$ and thus established one of the most useful recent theorems in the theory of substitutions. Since the order of each operator in the regular group is a divisor of $n$ while the order of every other operator divides $n-1$ it follows that there can be only one subgroup of order $n$.

During the last four years the enumerations of intransitive, imprimitive, and primitive groups have each been extended to one larger degree. The list of the intransitive groups of degree eleven includes 1,492 distinct substitution groups, which is about 500 more than the number for degree $10 . \S$ Nothing seems to have been done towards giving general formulas by means of which the number of these groups can be determined without trial except for the cases when the order is either the product of two distinct primes or when it is 4. Miss Martin published a list of the imprimitive groups of degree 15 and also one of the primitive groups of degree 18. \| She remarks that Dr. Kuhn has also made a study of the imprimitive groups of degree 15 and has arrived at results which differ greatly from those which she obtained.

'The efforts to discover a simple group of odd composite order have led to the study of primitive groups of such an order, for every simple group is simple isomorphic with one or more primitive substitution groups. Burnside proved the interesting theorem that the maximal subgroup which does not involve one of the elements of such a group must contain an even number of transitive constituents. 1 Dr. Rietz has also established a number of theorems relating to this maximal subgroup** which seems to present one of the most accessible approaches towards

* Vol. 25 (1897), p. 16 ; vol. 26 (1898), p. 249.

† Vol. 32 (1901), p. 240.

$\ddagger$ Frobenius, Berliner Sitzungsberichte, 1901, p. 1220.

\&. Miller and Ling, Quar. Jour. of Math., vol. 32 (1901), p. 342.

|| Miss Martin, Amer. Jour. of Math., vol. 23 (1901), p. 259.

I Burnside, Proc. of the London Math. Soc., vol. 33 (1901), p. 165.

** Bulletin, vol. 8, pp. 17 and 275. 
the theory of primitive groups. By means of these theorems and the important theorem, due to Burnside, that there is no primitive group of odd composite order and of a prime degree $p$ that contains more than one subgroup of order $p$, Dr. Rietz has determined all the primitive groups of odd order whose degree does not exceed 242. All these groups are solvable.

It is well known that the average number of elements in all the substitutions of a transitive group of degree $n$ is $n-1$. Hence every transitive group must contain at least $n-1$ substitutions of degree $n$ to make up the average for the identity. In general, for each of its substitutions of degree $n-\alpha$ there must be $\alpha-1$ substitutions of degree $n$. Dr. Rietz has recently proved * a closely related theorem to the effect that a primitive group of degree $n$ and of order $g$ contains more than $g / x+1$ substitutions of degree less than $n, x$ being the number of transitive constituents in a maximal subgroup of degree $n-1$. This theorem is closely related to the investigations of Jordan, Bochert, Maillet, and others in reference to the class of a primitive group.

Although the primitive groups of low classes present much greater difficulties than those of low degrees, yet it is easy to determine all the primitive groups of a prime class $p$ while no one has yet succeeded to determine those of any general degree. $\dagger$ In a memoir published in Videnskabs-Selskabets Skrifter, 1897, Sylow considers the groups of degree $p$ and of order $p(p+1) \pi, \pi$ being a divisor of $p-1$. He proved that there are no groups of degree $p$ and of order $p / 2\left(p^{2}-1\right)$ except in the cases when $p$ has one of the values $5,7,11$. In each of these special cases there is only one such group.

Lombardi has recently developed a number of theorems in regard to imprimitive groups. $\ddagger$ Although his results differ but slightly from well-known theorems yet no references are given. Dr. Kuhn has made a much more extensive study of the theory of these groups. Only abstracts of his memoir have appeared.§ In the first of these it is pointed out that there are groups in addition to those noted by Dyck in Mathematische Annalen, volume 22, which cannot be represented in any non-regular imprimitive form. In connection with Wilkinson's article in regard to Jordan's theorem on the constancy of the factors of

\footnotetext{
* Bulletin, vol. 8, p. 276.

† Jordan, Liouville, vol. 17 (1872), p. 363.

† Lombardi, Giornale di Mathematiche, vol. 39 (1901), p. 134.

$\S$ Bulletin, vol. 7 (1901), pp. 5 and 116.
} 
imprimitivity* it should be observed that Jordan himself noted the error in his Traité des Substitutions. He published a note in regard to the matter in Giornale di Matematiche, volume 10 (1872), page 116.

An abstract group is generally said to be represented by a substitution group if the two groups are simply isomorphic. The term is used in this sense when it is said that every abstract group can be represented as a regular substitution group. Quite recently Burnside published an article in which he uses this term in a more general sense in accord with the phraseology used by Frobenius in the study of linear substitutions. Any substitution group to which an abstract group has an $(\alpha, 1)$ isomorphism is said to be a representation of the abstract group. $\dagger$

According to this use of the term $1,(a b)$ is a representation of every group which involves a subgroup of half its order. It appears to the writer somewhat unfortunate to use a term which had such definite meaning in the theory of substitutions, in this general way. It may be added that some of the statements of this article are not in accord with known results unless other terms are also given an unusual meaning. Lines 8-11 on page 161 may serve as an instance. Additional references to other articles bearing on the same question would have increased the usefulness of the article very materially.

Beginners in the study of the theory of substitutions are naturally much hampered by the different uses of the same term as well as by the number of different terms employed to express the same concept. Within the last few years Netto has perhaps made the most serious contribution towards increasing the latter difficulties by using such new terms as conjug, conjuge complexe, and autojugen Theiler. The last of these terms is used for invariant subgroup so that the Germans can now express this concept by any one of the following six terms : invariante, ausgezeichnete, or monotypische Untergruppe ; autojuger, eigentlicher, or Normaltheiler. The first four and the last of these seem to be due to König, Lie, Frobenius, Netto, and Weber respectively, while eigentlicher Theiler seems to have been derived from décomposition propre, used by Galois $§$ who introduced the concept of invariant subgroup.

* Wilkinson, Quar. Jour. of Math., vol. 30 (1898), p. 157.

† Burnside, Proc. of the London Math. Soc., vol. 34 (1902), p. 159.

† Netto, Vorlesungen über Algehra, 1900, p. 328.

\& Liouville, vol. 11 (1846), p. 408. 
Frobenius has employed the terms inner and outer automorphisms * instead of Hölder's terms cogredient and contragredient isomorphisms. These new terms have the advantage of being shorter and perhaps more natural than the older ones. Moreover, it seems desirable to have a term which explicitly states that a simple isomorphism is under consideration. This may be done by either of the new terms holomorphism or automorphism, which were introduced about the same time. Possibly it would be well to restrict the latter, as Frobenius has done, to express a simple isomorphism of a group with itself, while the former might express a general simple isomorphism.

It may be observed that the nomenclature here suggested is not free from objections. It is sometimes desirable to speak of a general isomorphism of a group with itself, and it would not appear unnatural to use the term automorphism for this purpose instead of restricting its use to a simple isomorphism of a group with itself. Moreover, Ritter, at the suggestion of Klein, has employed holomorphism for a general isomorphism and has used the term isomorphism to represent only a simple isomorphism. Burkhardt has followed this terminology in his Encyklopädie article. $\dagger$ It is open to the objection that the term isomorphism has been extensively used in a broader sense.

As the elements of a substitution group are not, in general, numbers, their laws of combination cannot be directly represented by relations between numbers. In a series of articles published in the Mathematische Annalen and in Crelle, Hoyer has developed a new theory for the algebraic solution of the problems of substitution groups. $\neq$ These developments not only concern themselves with abstract group properties, such as the smallest number of generational relations and the finiteness of the order, but also with those relating to the representation as substitution groups, such as the degree of transitivity, the class, and the systems of imprimitivity. The general question of the determination of all the possible substitution groups of degree $n$ is reduced to an algebraic one. Most of the developments are quite general and the results do not appear directly useful in advancing the theory along the older lines.

\footnotetext{
* Frobenius, Berliner Sitzungsberichte, 1901, p. 1324.

† Burkhardt, Encyklopädie der Mathematischen Wissenschaften, vol. 1, p. 220.

$\ddagger$ Hover, Math. Annalen, vol. 49 (1897), p. 39 ; vol. 50 (1898), p. 499 ; vol. 51 (1899), p. 445 ; vol. 52 (1899), p. 550 ; Crelle, vol. 124 (1901), p. 102.
} 


\section{§4. Group Characteristics.}

The independent generators of any abelian group $A$ may be represented by cyclic substitutions in distinct sets of letters. If the orders of these cyclic substitutions are $\rho_{1}, \rho_{2}, \cdots, \rho_{m}$ the order of $A$ is $\rho_{1} \rho_{2} \cdots \rho_{m}$. The group generated by any one of these substitutions of order $\rho_{a}$ is simply isomorphic with the $\rho_{a}$ roots of unity. If the roots which may be associated with the different generating substitutions are regarded as independent numbers just as the substitutions are independent, these roots will generate a group which is simply isomorphic with $A$. The numbers which constitute the operators of this group are the group characteristics of $A$.

A more abstract definition of the characteristics of $A$ is as follows:* With every operator $s$ of $A$ there is associated a number which may be regarded as a function of $s$ and is denoted by $x(s)$. This number must satisfy the following conditions: (1) It does not vanish for any value of $s,(2)$ the equation $x\left(s s^{\prime}\right)=x(s) x\left(s^{\prime}\right)$ is satisfied for every pair of operators in $A$. Two such numbers as $x, x^{\prime}$ are to be regarded as different when there is some operator $s_{1}$ in $A$ such that $x\left(s_{1}\right) \neq x^{\prime}\left(s_{1}\right)$. The numbers thus defined are the group characteristics of $A$ and it is not difficult to deduce the properties mentioned in the preceding paragraph from these more abstract definitions. The characteristics of an abelian group were already employed by Lagrange and Dirichlet $\dagger$ and their abstract definition is due to Dedekind.

Since 1896 Frobenius has published a series of mernoirs in which he has developed a theory of group characteristics for non-abelian groups, which reduces to the preceding when the group is abelian. $\div$ He proves that the number of distinct characteristics is equal to the number of complete sets of conjugate operators of a group and that a characteristic of a given operator is the same as that of each of its conjugates. Moreover, each of the characteristics is the sum of roots of unity and the product of two characteristics can be represented as a linear function of all the characteristics of the group.

Weber gives a brief exposition of the theory of characteristics in the second edition of his Algebra, volume 2, 1899. More

\footnotetext{
* Cf. Weber, Lehrbuch der Algebra, vol. 2 (1899), p. 49.

$\dagger$ Frobenius, Berliner Sitzungsberichte, 1896, p. 985.

$\ddagger$ Loc. cit., 1896 , pp. 985,$1343 ; 1897$, p. $994 ; 1898$, p. $501 ; 1899$, pp. 330 and $482 ; 1900$, pp. 303 and 516.
} 
recently, Burnside has developed the same subject from a somewhat different standpoint in an article published in the Proceedings of the London Mathematical Society.* In two subsequent articles published in the same volume he has applied the theory in the study of some properties of groups of odd order. In the first of these papers he proves that a linear substitution of odd order is reducible whenever all the coefficients are real and that there is no simple group of an odd composite order and of a prime degree. The main result arrived at in the second of these papers is that if an odd prime $p$ is the smallest factor of the order of a group there must be an invariant subgroup of index $p$ unless either $p^{4}$ or $p^{3} q$, where $q$ is a prime factor of $p^{2}+p+1$, is a factor of the order.

To facilitate the application of characteristics Frobenius developed two methods which are more convenient in many special cases than the general method developed in 1896. The first of these is based upon the relations which exist between the characteristics of a group and those of a subgroup. $\dagger$ The case when this subgroup is invariant leads to especially interesting results. Moreover, a characteristic can be directly obtained by this method in case the group is represented as a doubly transitive substitution group. The second method is based upon the composition of the characteristics and is illustrated by means of the alternating and the symmetric groups of degree 6 as well as by the tetrahedral, octahedral and icosahedral rotation groups. $\ddagger$

The Berliner Sitzungsberichte for 1901 contains three papers by Frobenius on solvable groups. In the first two of these group characteristics are extensively used. The main theorem of the first paper is as follows: If a group of order $f g, f$ and $g$ being relatively prime, contains a subgroup of order $f$ composed of invariant operators then it must be the direct product of this subgroup and a subgroup of order $g$. This theorem was afterwards proved without the use of group characteristics by de Seguier in Comptes Rendus, volume 134 (1902). The second of these papers contains, among many others, the interesting theorem mentioned in the preceding paragraph that a group of class $n-1$ always contains a regular group of order $n$.

The principal theorem which is proved in the last of these three articles may be stated as follows : If $p^{\lambda}$ is the highest power

* Vol. 33 (1901), p. 146.

† Frobenius, Berliner Sitzungsberichte, 1898, p. 501.

$\ddagger$ Loc. cit., 1899 , p. 330 . 
of $p$ which divides the order of a group $H$, and if no operator of $H$ whose order is prime to $p$ transforms a subgroup of order $p^{\mu}$ into itself without being commutative with each one of its operators, then must $H$ contain a subgroup of index $p^{\lambda}$ which is composed of all the operators of $H$ whose orders are not divisible by $p$. Frobenius observes that it is possible to state this theorem somewhat more generally; but in this case the statement becomes still more complex and we shall not present it here.

STANFORD UNIVERSITY, June, 1902.

\section{SHORTER NOTICES.}

Urkunden zur Geschichte der Mathematik im Mittelalter und der Renaissance. By M. CurTze. Erster Theil. Abhandlungen zur Geschichte der mathematischen Wissenschaften, XII. Heft. Leipzig, Teubner, 1902. 336 pp. 16 Marks.

IT is a compliment to the monumental work of Professor M. Cantor that the activity in the field of the history of mathematics for the past twenty years has been almost entirely directed by him. The sole effort has been to supplement his work, to enter some of the innumerable doors which he has opened, to decipher the inscriptions upon the monument which he has erected. Hardly an article appears in the Bibliotheca Mathematica, relating to the period preceding the middle of the eighteenth century, that does not refer in some way to Cantor's work, and the Abhandlungen have been more or less under his direction for a quarter of a century.

Herr Curtze's latest contribution is an evidence in point, not merely in being dedicated to Professor Cantor on the occasion of his doctor's jubilee, but in that it elaborates certain details of his History for which elaboration scholars have been waiting.

Half of the work is given to the Liber embadorum of Abraham Savasorda (Sahib al Schorta, chief of the guards) as translated from the Hebrew by Plato of Tivoli in 1116, a treatise merely mentioned by Cantor.* The work has already been noticed by Curtze $\dagger$ as being one of the chief sources of

* Vorlesungen, vol. 2, p. 853.

† Bibliotheca Mathematica, vol. 1, 3d series (1900), p. 501. 